\title{
Erratum
}

\section{A Nonconforming Combined Method for Solving Laplace's Boundary Value Problems with Singularities}

\section{Zi-CAi Li}

Department of Computer Science, Concordia University, 1455 de Maisonneuve Blvd. West, Montreal, Qucbec H3G 1M8, Canada

Numer. Math. 49, 475-497 (1986)

On page 481 in Lemma 1 and page 483 in Theorem 2, the assumption Meas $\left(\overline{A B} \cap \hat{S}_{1}^{h}\right) \neq 0$ should be changed to Meas $\left(\overrightarrow{A C} \cap \hat{S}_{1}^{h}\right) \neq 0$. Therefore, in the proof procedure, at line 20 of page $481, \operatorname{Meas}(\overrightarrow{A B}$ $\left.\cap S_{2}\right) \neq 0$ should read as Meas $\left(\overline{A C} \cap S_{2}\right) \neq 0$, and at line 9 of page 483 , Meas $\left(\overline{A B} \cap S^{h 1}\right) \neq 0$ should read as Meas $\left(\overline{A, C} \cap \hat{S}_{1}^{h} \neq 0\right.$. Besides at line 16 of page $483, \int_{R^{*}}$ should be $\int_{l_{R^{*}}}$. 